\title{
Comparison of Spray and Point Inoculation with Fusarium graminearum to Evaluate FHB Disease in Two Winter Wheat Genotypes Under Temperature Stress
}

\author{
Victor Chijioke Okereke \\ Department of Crop and Soil Science, University of Port Harcourt, Port Harcourt, Nigeria
}

Email address:

chykeoky@yahoo.com

\section{To cite this article:}

Victor Chijioke Okereke. Comparison of Spray and Point Inoculation with Fusarium graminearum to Evaluate FHB Disease in Two Winter Wheat Genotypes Under Temperature Stress. Computational Biology and Bioinformatics. Vol. 5, No. 1, 2017, pp. 1-5.

doi: 10.11648/j.cbb.20170501.11

Received: August 10, 2016; Accepted: August 24, 2016; Published: April 13, 2017

\begin{abstract}
Two near isogenic lines of wheat; Mercia 1 (Rht-B1b) and Mercia 2 (Rht-D1b) were evaluated for Type I and Type II resistance using point or spray inoculation and incubated under controlled environment. The experiment was a complete factorial combination of $2 \times 4 \times 2 \times 4\{2$ genotypes, 4 inoculation treatments (spray + Fusarium, spray + SDW, point + Fusarium, point + SDW) $\times 2$ temperatures $\}$ and 4 randomised replicates. Wheat spikes were sprayed with a single spore isolate of $F$. graminearum 4 days after the start of flowering and transferred to controlled environment cabinets set at either $23 / 15^{\circ} \mathrm{C}$ or $28 / 20^{\circ} \mathrm{C}$ for 14 days and then taken outside to mature. Results reveals that genotype showed no difference in FHB severity in both spray inoculation and point inoculation but the temperature main effect only approached significance $(\mathrm{P}=0.071)$ with low temperature increasing $\mathrm{FHB}$ severity following point inoculation. High temperature significantly $(\mathrm{P}<0.001)$ increased DON concentration in spray inoculation, contrasted the effect in point inoculation. However, the amount of DON per grain showed no significant $(\mathrm{P}>0.05)$ effect. Grains per spike was significantly $(\mathrm{P}<0.011)$ reduced by $25 \%$ following spray when compared with point inoculation, but showed no significant $(\mathrm{P}>0.05)$ effect of temperature. On average, Fusarium infection significantly $(\mathrm{P}<0.001)$ reduced wheat grain weight by $28 \%$ when compared with uninoculated control.
\end{abstract}

Keywords: Point, Spray, Inoculation, Mercia 1, Mercia 2, Fusarium graminearum, Type 1 Resistance, Type 2 Resistance

\section{Introduction}

Two major dwarfing alleles Rht-B1b and Rht-D1b formerly known as Rht1 and Rht2 respectively, derived from Norin 10 have been used in over half the World's wheat crop [1]. In the UK, the majority of recommended wheat cultivars contain Rht-D1b after its first introduction in 1974 [2] and wheat cultivars carrying Rht-D1b have been linked to higher susceptibility to FHB. This is possibly due to the shortened distance from the spike to infected crop debris [3]. The resistance of wheat to FHB is a complex phenomenon due to different factors that are involved in the infection [4]. According to [5], cultivars differ with the growth stage at which they are most susceptible. However, of most importance in susceptible genotypes is the likelihood of mycotoxin production of which deoxynivalenol (DON) is of most concern and in highly resistant genotypes resistance is the major factor in suppressing disease development and DON accumulation. [3] categorized the types or components of resistance as follows: Type I (resistance to initial infection); Type II (resistance to spread within the spike); Type III (resistance to kernel infection); Type IV (tolerance infection) and Type V (resistance to DON accumulation). Different inoculation methods have been developed by researchers to distinguish between these sources of resistance. However, spray inoculation covers both Type I and Type II resistances, while inoculation of spores in a single floret per head (point inoculation) estimates Type II resistance [6, 7]. Point inoculation in FHB evaluation is a relatively stable method and is less affected by environment in terms of disease development [8]. [5] illustrated that some wheat lines could have both Type I and Type II resistance present while others could have only one type of resistance. Greenhouse evaluation has mostly employed the point inoculation methods whereas 
for large scale breeding program, spray inoculation are mainly used $[9,10] .[11]$ in their findings observed that Type I and Type II resistance vary independently. Some authors [7, 9] have identified the environment and interaction between genotype and environment as important factors that influence the method of inoculation.

As reported [12], screening for Type II resistance by point inoculation is most consistent for assessing FHB severity under controlled environmental conditions. It will therefore be of interest to examine whether both inoculation methods will maintain the same genotype ranking against FHB under a small change in temperature as predicted under climate change. This study was therefore aimed at comparing the expression of FHB infection after spray and point inoculation under temperature stress using two near isogenic lines (NILs) of Mercia background differing in FHB resistance.

\section{Materials and Methods}

Near isogenic lines of Mercia 1 (Rht-B1b) and Mercia 2 (Rht-D1b) were compared using spray and point inoculation of $F$. graminearum to evaluate tolerance to FHB under temperature stress. Seeds were grown in 12.5 -cm-diameter pots filled with a 4:4:2:1 mixture of steam-sterilized $6 \mathrm{~mm}$ gravel, medium vermiculite, and $3 \mathrm{~mm}$ sharp sand and peatbased potting compost. To supplement plant nutrition, $2 \mathrm{~kg}$ of Osmocote Pro 3-4 months (Scotts, UK) was added per cubic metre of planting mixture. Pots filled with planting medium were soaked overnight and five seeds of the different cultivars or genotypes were sown on each pot at a depth of 2$2.5 \mathrm{~cm}$ and then thinned to three per pot at the three leaf stage. Pots were then transferred outside and raised to a height of approximately $10 \mathrm{~cm}$ on bricks to allow free water drainage. Pots were irrigated automatically through a drip irrigation system twice daily. At the start of booting \{Growth Stage (GS); [13] first tillers were tagged so that they could be identified spore inoculation. The plants were treated for powdery mildew 82 days after sowing with Flexity $(300 \mathrm{~g} / \mathrm{litre}(25.2 \% \mathrm{w} / \mathrm{w})$ metrafenone; BASF Plc, UK) at $0.51 /$ ha. Fusarium inoculation was done at 4 days after start of anthesis followed by heat treatment a day after. The experiment was a complete factorial combination of $2 \times 4 \times 2$ $\mathrm{x} 4\{2$ genotypes, 4 inoculation treatments (spray + Fusarium, spray + SDW, point + Fusarium, point + SDW) $\mathrm{x}$ 2 temperatures and 4 randomised replicates $\}$.

For the spray inoculation, the first tillers were sprayed with $1 \mathrm{ml}$ of $1 \times 10^{5} / \mathrm{ml}$ spore suspension per ear using a hand sprayer, while point inoculation used $1 \mathrm{ml}$ of the Fusarium spore suspension pipetted between the lemma and palea of the middle floret. The corresponding control plants were also treated accordingly using sterile distilled water. After inoculation, both the inoculated and controls plants were enclosed for 24 hours using clear polythene bags to increase humidity and promote disease development and plants subjected to heat stress treatment as earlier described. Following spray inoculation, disease severity was measured as the percentage of infected spikelet per spike while following point inoculation; disease was measured as the proportion of diseased spikelets per spike and relating them to total number of spikelets of the respective spike [7]. Harvesting was done when the plants were fully senesced and the grain below $15 \%$ moisture content. The spikes were hand threshed carefully to avoid the loss of infected and shrivelled kernels. From these kernels, different yield parameters were determined. Total grain number per spike was evaluated by counting the number of grains per spike before separating the damaged kernels and expressing as a percentage. Mean grain weight was evaluated by weighing all grains in each spike and dividing by the total number of grains and grain yield evaluated as the total weight of the healthy grains. DON concentration of the extract was analysed using Enzyme Linked Immunosorbent Assay (ELISA) DON kits (Agra Quant, Romer Labs, Singapore Pte Ltd) according to the manufacturer's instructions. The DON range of quantification was between $0.25-5.0 \mu \mathrm{g} / \mathrm{g}$. The treatments were completely randomised in the micro titre plates. Absorbance was measured at $450 \mathrm{~nm}$ and a differential filter of $630 \mathrm{~nm}$ using a Multiskan Ascent plate reader. DON concentration was calculated by reference to a standard curve generated using the DON kit. All statistical analyses were carried out using GenStat (GenStat ${ }^{\circledR}$ 13th Edition, VSN International Ltd., UK). An analysis of variance (ANOVA) was carried out to determine the effects of the treatments on FHB severity, FDK, DON concentration, mean grain weight and yield. Data did not require transformation as they were normally distributed.

\section{Results}

Results obtained showed that genotype did not defer in disease severity in both spray and point inoculation but the temperature main effect approached significance $(\mathrm{P}=0.071)$ (Fig. 1) only in point inoculation; with low temperature increasing FHB severity. Conversely, both methods resulted in similar disease severity in terms of percent infected spikelets. There was significant $(\mathrm{P}=0.05)$ genotypic difference occurring in FDK with Mercia 2 having higher percentage of FDK (Fig. 2). Following spray and point inoculation, DON concentration showed no significant $(\mathrm{P}>0.05)$ main effect of genotype, inoculation method or temperature, but the temperature $\mathrm{x}$ method of inoculation was significant $(\mathrm{P}<0.001)$ (Fig. 3). Low temperature increased DON concentration in point inoculation while the DON concentration in spray pots was higher at high temperature. However, both point and spray inoculation resulted in a similar grain DON content in the harvest grains regardless of the genotype or incubation temperature (Fig. 4a $\& \mathrm{~b})$. A significant effect $(\mathrm{P}<0.011)$ of method of inoculation was detected in the grains per spike. Regardless of the genotype and incubation temperature, spray inoculation reduced the number of grains per spike by $25 \%$ compared to point inoculation (Fig. 5). When compared with the uninoculated control, higher grain reduction was obtained under spray inoculation (38\%) than point inoculation (20\%). Mean grain weight showed no significant $(\mathrm{P}>0.05)$ effect of 
temperature, genotype or method of inoculation, but on average, Fusarium infection significantly $(\mathrm{P}<0.001)$ reduced grain weight by $28 \%$ (Fig. 6).

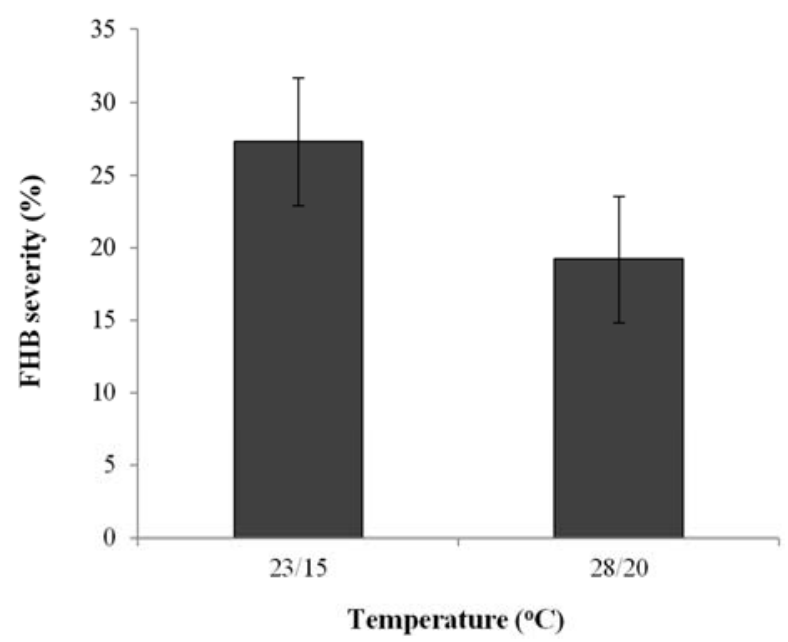

Figure 1. Effect of temperature applied at $G L+5$ on FHB severity of two winter wheat genotypes; Mercia 1 and Mercia 2 following point inoculation with F. graminearum and maintained in controlled growth conditions.

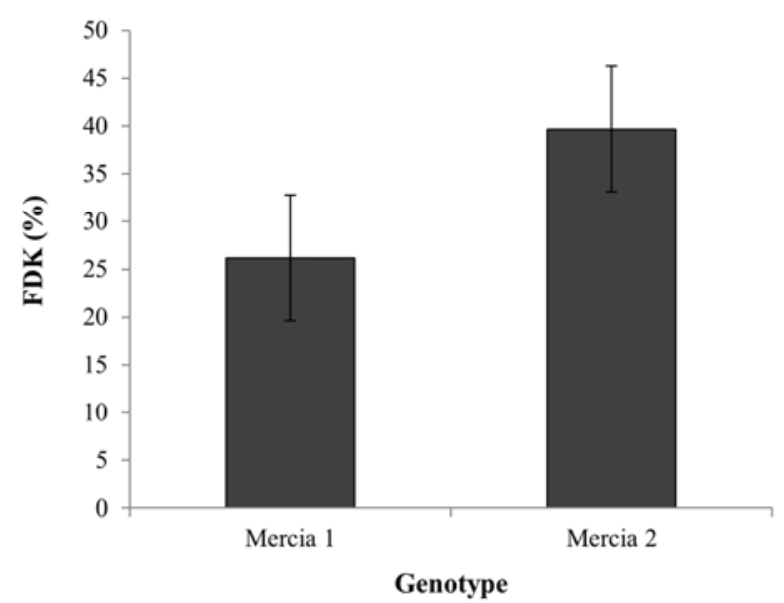

Figure 2. Percentage Fusarium damaged kernel of two winter wheat genotypes; following point and spray inoculation with F. graminearum and maintained at either $23 / 15^{\circ} \mathrm{C}$ or $28 / 20^{\circ} \mathrm{C}$ in controlled environment.

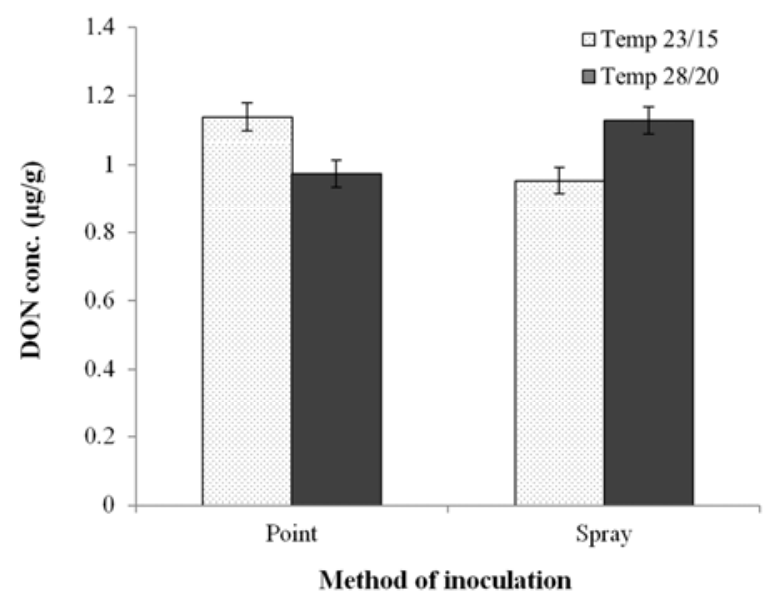

Figure 3. Effect of temperature applied at GL+5 on DON concentration of two winter wheat genotypes; following point and spray inoculation with $F$. graminearum and maintained in controlled environment.
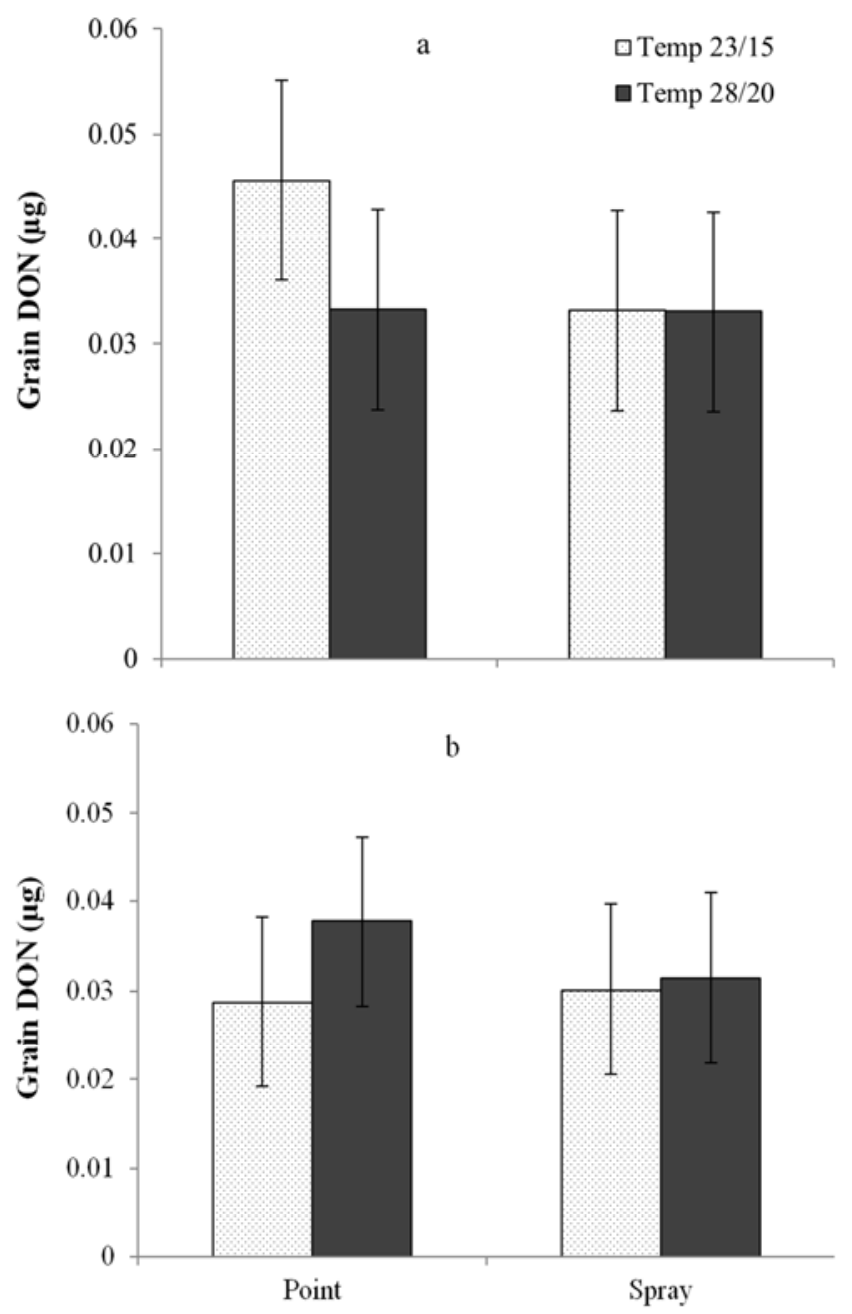

Method of inoculation

Figure 4. Effects of temperature and method of inoculation on grain DON content of two winter wheat genotypes (a) Mercia 1 and (b) Mercia 2. Wheat spikes were either spray or point inoculated with $F$. graminearum and maintained in controlled environment.

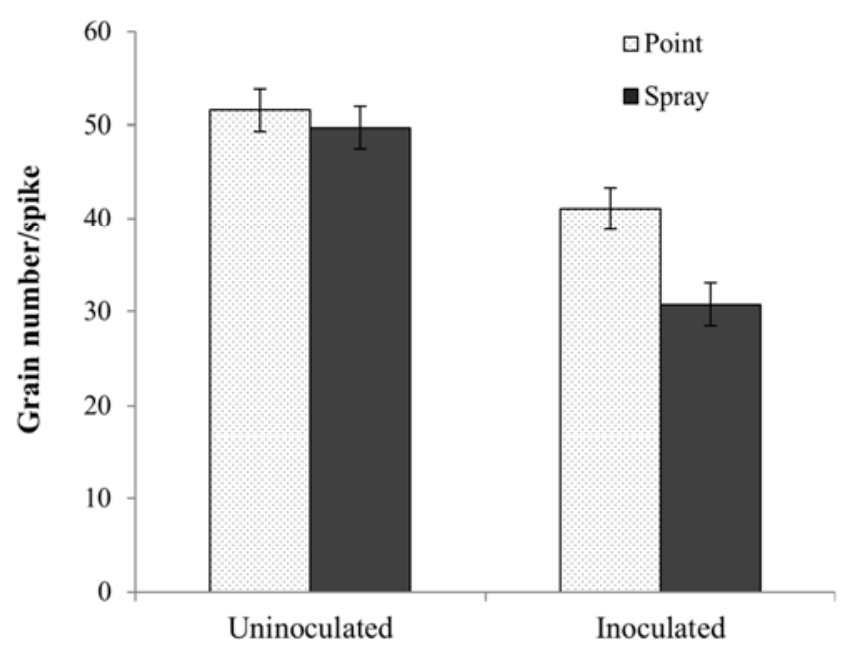

Figure 5. Effect of method of inoculation on number of grains per spike of winter wheat genotypes; Mercia 1 and Mercia 2 following inoculation with F. graminearum and SDW and maintained at either $23 / 15^{\circ} \mathrm{C}$ or $28 / 20^{\circ} \mathrm{C}$ in controlled environment. 


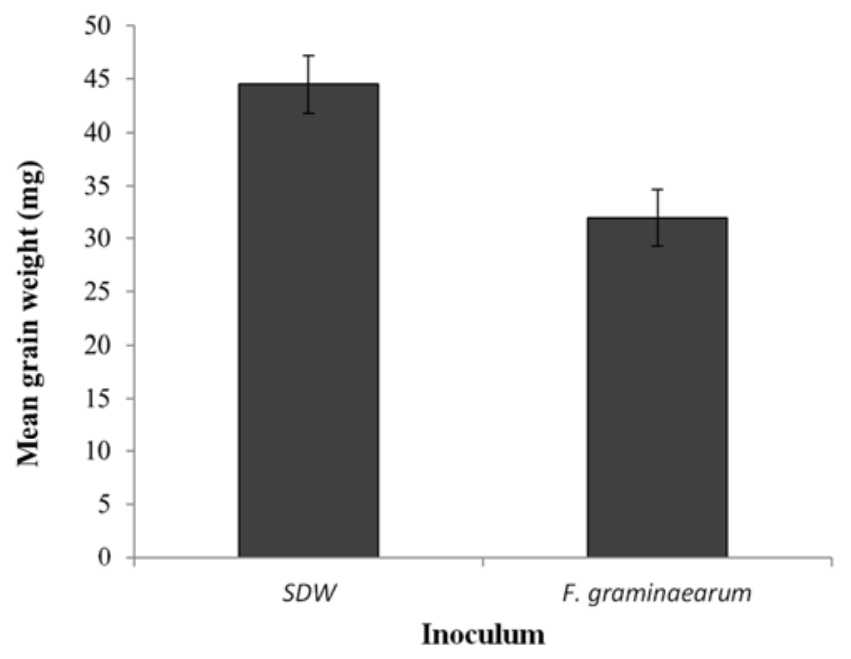

Figure 6. Mean effects of inoculum on mean grain weight of two winter wheat genotypes; Mercia 1 and Mercia 2, following point and spray inoculation and maintained at either $23 / 15^{\circ} \mathrm{C}$ or $28 / 20^{\circ} \mathrm{C}$ in controlled environment.

\section{Discussion}

Results of evaluation of Type 1 and Type 11 resistance in the current study is consistent with other findings that midanthesis is the most susceptible stage and temperature may play significant impact on disease severity. An addition to these findings was the possible effect of incubation temperature of $23 / 15^{\circ} \mathrm{C}$ on DON concentration following point inoculation. Whilst [9] reported that Type I and Type II resistance might be governed by different loci which measure different resistance reactions, [6, 14] associated major resistance QTL for low FHB severity with low DON content in wheat and barley. Longer duration of flower opening experienced under low temperature might explain the higher DON in point inoculation. Previous experiments showed no temperature difference at inoculation at mid-anthesis, so the difference observed in this experiment could be attributed to the timing of temperature stress and may also be responsible for the genotypic difference found in FDK. Stressing the plants a day after inoculation may have had greater influence on spray inoculation and particularly Rht-D1b; unlike in the previous studies where pots were transferred into growth cabinets days after inoculation. FDK is very important in wheat breeding programmes as this is normally the preferred parameter when evaluating wheat breeding lines for FHB resistance [15]. As observed by [9], no genotype combines different levels of both Type I and Type II resistance and often the differences between both types of resistance are marginally significant. This may explain the non-significant genotype $\mathrm{x}$ method of inoculation interaction observed in all the parameters. Number of grains per spike, grain weight and grain yield were neither affected by genotype or temperature but strongly influenced by the method of inoculation. This suggests that the method of inoculation can be considered to be more stable than either the incubation temperature or genotype, as it affected the different parameters measured. The higher grains lost recorded following spray inoculation was expected and the reduction in grain weight which was independent of the method of inoculation shows the importance of both inoculation methods in FHB assessment. Grain weight reduction as a result of FHB infection has been found to relate negatively with FDK [16], and this is a strong estimator of the presence of mycotoxin in wheat grains.

\section{Conclusion}

The susceptibility of wheat genotypes to Fusarium head blight at 4 days after anthesis (mid-anthesis) regardless of the associated dwarfing allele is an important factor in FHB infection and was also confirmed in this work. Evaluation of Type I and Type II resistance showed that Rht-B1b and RhtD1b did not differ in their susceptibility to DON accumulation, but that the mechanism of resistance could be influenced by temperature. Genotypic difference was only observed in FDK and reemphasized the importance of FDK in FHB rating. However, the observation of the stability of Type II resistance under low temperature needs further investigations to confirm whether the adverse effect of low temperature as observed in the current study could be replicated.

\section{References}

[1] Miedaner, T., Voss, H. H. 2008. Effect of dwarfing Rht genes on Fusarium head blight resistance in two sets of nearisogenic lines of wheat and check cultivars. Crop Science 48: 2115-2122.

[2] Gosman, N., Bayles, R., Jennings, P., Kirby, J., Nicholson, P. 2007. Evaluation and characterization of resistance to Fusarium head blight caused by Fusarium culmorum in UK winter wheat cultivars. Plant Pathology 56: 264-276.

[3] Mesterhazy, A. 1995. Types and components of resistance to Fusarium head blight of wheat. Plant Breeding 114: 377-386.

[4] Stack, R. W. 2000. Return of an old problem: Fusarium head blight of small grains. Plant Health Review, June 2000.

[5] Schroeder, H. W., Christensen, J. J. 1963. Factors affecting resistance of wheat to scab caused by Gibberella zeae. Phytopathology 53: 831-838.

[6] Bai, G. H., Plattner, R., Desjardins, A., Kolb, F. S., Jones, S. 2001. Resistance to Fusarium head blight and deoxynivalenol accumulation in wheat. Plant Breeding 120: 1-6.

[7] Miedaner, T., Moldovan, M., Ittu, M. 2003. Comparison of spray and point inoculation to assess resistance to Fusarium head blight in a multi environment wheat trial. Phytopathology 93: 1068-1072.

[8] Malbrán, I., Mourelos, C. A., Girotti, J. R., Aulicino, M. B., Balatti, P. A., Lori, G. A. 2012. Aggressiveness variation of Fusarium graminearum isolates from Argentina following point inoculation of field grown wheat spikes. Crop Protection 42: 234-243.

[9] Miedaner, T. 1997. Breeding wheat and rye for resistance to Fusarium diseases. Plant Breeding 116: 201-220. 
[10] Rudd, J. C., Horsley, R. D., McKendry, A. L. and Elias, E. M. J. C. 2001. Host Plant Resistance Genes for Fusarium Head Blight: Sources, Mechanisms and Utility in Conventional Breeding Systems. Crop Science 41: 620-627.

[11] Desmeules, J., Paulitz, T., Rioux, S., O'Donoughue, L., Mather, D. 2001. Fusarium head blight symptom development in spring what genotypes after inoculation with point and spray methods. Proceeding of Canadian Workshop on Fusarium head blight. Ottawa, November 3-5, Pp 50.

[12] Bai, G., Shaner, G. 1994. Scab of wheat: Prospect for control. Plant Disease 78: 760-766.13.

[13] Zadoks, J. C., Chang, T. T, Konza, C. F. 1974. A decimal code for the growth stages of cereals. Weed Research 14: 415-421.
[14] Bai, G., Shaner, G. 2004. Management and resistance in wheat and barley to Fusarium head blight. Annual Review of Phytopathology 42: 135-161.

[15] Thompson, C. J., 2010. Evaluation of an inoculation method and quantitative trait loci for Fusarium head blight resistance in wheat. Unpublished Master of Science Thesis, University of Illinois at Urbana-Champaign.

[16] Mesterházy, A., Bartok, T., Kaszonyi G., Varga, M., Toth, B., Varga., J. 2005. Common resistance to different Fusarium spp. Causing Fusarium head blight in wheat. European Journal of Plant Pathology 112: 267-281. 